\title{
ÚLCERA VARICOSA Y CIRUGÍA DE VÁRICES ¿CÓMO NOS VA?
}

Juan Fernando Muñoz MD*, Luís Eduardo Mendoza MD*, Oswaldo Ceballos MD **, José Mercado Rosa MD***

\section{Resumen}

describir las características de los pacientes con úlcera venosa llevados a cirugía para varicosafenectomía en el Hospital de San José de enero 1 a diciembre 31 de 2006. Métodos: se consideraron 23 pacientes con UV activa que fueron llevados a varicosafenectomía en el año 2006 en el Hospital de San José; se tomó la información de las variables de las historias clínicas, se tabularon en una hoja Excel realizando tablas de frecuencias y análisis bivariado. Resultados: cicatrización de la ulcera en 100\% con un tiempo promedio de 1.5 meses (0.59) y seguimiento promedio de 2.84 meses. No se encontró diferencia entre las variables analizadas y el tiempo de cicatrización. Conclusión: con el presente estudio podemos reafirmar la utilidad de la cirugía para el manejo de la UV cuando la causa es insuficiencia venosa superficial.

úlcera varicosa, insuficiencia venosa superficial, varicosafenectomía.

UV, úlcera varicosa; IVC, insuficiencia venosa crónica; CEAP, clínico, etiología, anatomía y patología.

\section{Abstract}

Objective: to describe the features of patients with venous ulcers (VU) who underwent a long saphenous vein stripping (LSVS) between January 1 and December 31, 2006 in San José Hospital. Methods: the trial includes 23 patients with active VU who underwent LSVS during 2006 in San José Hospital; data on variables was obtained from clinical records, tabulated in Excel sheets using frequency tables and bivariate analyses. Results: ulcer healing was obtained in $100 \%$ of patients, in a median time of 1.5 months (0.59) and a mean follow-up period of 2.84 months. No difference was found between analyzed variables and healing time. Conclusion: this study contributes to reassert the usefulness of LSVS to treat VU secondary to superficial venous insufficiency.

varicose ulcer, superficial venous insufficiency, long saphenous vein stripping (LSVS).

\section{Introducción}

Fecha recibido: noviembre I 4 de 2007-Fecha aceptado: enero I5 de 2008

* Coordinador programa de cirugía vascular periférica, Hospital de San José. Instructor Asociado, Fundación Universitaria de Ciencias de la Salud.

* Residente ll de cirugía vascular periférica, Hospital de San José. Fundación Universitaria de Ciencias de la Salud.

** Cirujano Hospital de San José. Profesor titular y jefe de la oficina de Planeación de la Fundación Universitaria de Ciencias de la Salud.

**** Cirujano general Hospital de San José, Instructor Asociado, Fundación Universitaria de Ciencias de la Salud.
Las várices son un problema de salud mundial que afecta en promedio a 76.1 por 100.000 habitantes por año en los Estados Unidos ${ }^{1}$ y la máxima expresión de ellas es la UV con una incidencia de 18.1 por 100.000 habitantes por año, observándose en el $4 \%$ de los mayores de 65 años de la población total. ${ }^{1,2}$ La UV es una complicación crónica de la enfermedad varicosa, como resultado de la IVC, por tanto cualquier persona que tenga várices y no reciba un 
tratamiento adecuado y oportuno, puede desarrollarla, empezando desde ese momento una serie de trastornos físicos, fisiológicos, sociales, familiares y hasta conyugales. En Estados Unidos se pierden 4.6 millones de días de trabajo por año atribuibles a las UV ${ }^{2}$ y el costo de la atención anual es de un billón de dólares. ${ }^{3}$

Mucho se ha escrito pero poco se ha logrado. Alrededor del año 200 a.c. en la India el médico Sushruta escribió en su libro Sushruta Samhita, el tratamiento de la UV que incluía desbridamiento, aplicaciones locales y vendajes inelásticos. ${ }^{4}$ En el siglo XVI de nuestra era el prestigioso médico Ambrosio Paré decía que las várices y las úlceras eran buenas. Afirmaba que las primeras servían para alejar de las partes nobles del cuerpo los humores y otras excreciones y que las úlceras eran la puerta de salida para la sangre mala. Durante siglos muchos médicos pensaban que las úlceras de las piernas no debían curarse porque era el sitio de drenaje de los humores nocivos y que si cicatrizaban causaban enfermedades. ${ }^{4}$

Los estudios sobre UV tradicionalmente se basan en comparar el manejo médico con la cirugía. Los resultados están en favor del último ya que con el manejo médico la cicatrización es del $50 \%$ en un tiempo promedio de seis meses y las recidivas son del 70 al $100 \%$ al año. ${ }^{5}$ Con el manejo quirúrgico hay reportes de curación del $85 \%$ de las úlceras a los seis meses, con un tiempo promedio de 1.5 meses y una recurrencia de $4.6 \%$ en cinco años de seguimiento. ${ }^{6}$

Sobre la formación de las úlceras hay muchas teorías. Sin embargo, lo que hoy se acepta es que el reflujo de la sangre en las venas de las piernas hace que se congestione la parte distal de las extremidades; este aumento de presión venosa termina lesionando el endotelio depositándose hemosiderina en el intersticio y liberando sustancias vasoactivas pro-inflamatorias y pro-coagulantes, potenciadas por la activación de los leucocitos que agrandan el proceso inflamatorio y terminan con la ulceración de la piel adyacente al evento inflamatorio. A pesar de todos los esfuerzos y de las mejores técnicas quirúrgicas, hay úlceras que se tardan mucho en cicatrizar o incluso que no lo hacen, llegando hasta $15 \%$ en las serie de Obermayer et al. ${ }^{6}$

La enfermedad varicosa afecta a las personas adultas, en especial mujeres, con una relación de $6: 1^{7} \mathrm{y}$ tiene una base genética condicionada por factores de riesgos establecidos como son: sobrepeso, estar mucho tiempo de pie, embarazos y consumo de anticonceptivos orales. Se acepta como insuficiencia venosa crónica (IVC) el conjunto de síntomas y signos que acompañan al funcionamiento anormal de una vena varicosa, como son cansancio, dolor, edema, pesadez, calambres y al progresar hay cambios en la piel y ulceración. Esta alterna con períodos de cicatrización cortos y recidivas de la úlcera en el $4.6 \%$ en cinco años. ${ }^{8}$

Para unificar la clasificación de la enfermedad varicosa en la década de los años 90 se hizo un consenso mundial adoptando la clasificación llamada CEAP que tiene en cuenta la clínica, etiología, anatomía y patología. Las clasifica en los siguientes estadios:

C1: telangiectasias, que son las "arañas vasculares", várices menores de un milímetro.

$\mathrm{C} 2$ :várices con dilataciones venosas mayores de $4 \mathrm{~mm}$.

C3: presencia de edema.

C4: con cambios en piel.

C5: cuando hay cicatrices de úlceras.

C6: con úlcera activa.

El diagnóstico se complementa con un dúplex color que permite analizar por medio de imágenes del flujo si hay obstrucción o reflujo, parámetros que determinan la elección de un tratamiento médico o quirúrgico. El primero consiste en mejorar los factores de riesgo (bajar de peso, suspender los anticonceptivos orales y hacer ejercicios) además de usar soporte elástico que contribuye a mejorar el retorno venoso, aliviando los síntomas y limitando la progresión de la enfermedad. Cuando no es suficiente, el tratamiento será quirúrgico. Consiste en retirar todas las venas lesionadas (varicosas) tanto del sistema superficial como las perforantes. 


\begin{tabular}{|c|c|c|c|}
\hline \multicolumn{4}{|c|}{ Tabla 3. Tratamiento previo } \\
\hline Cx previa & SI & NO & $\begin{array}{c}\text { SIN } \\
\text { DATOS }\end{array}$ \\
\hline $\begin{array}{c}\text { Vendas antes de } \\
\text { cX }\end{array}$ & $22(95.6)$ & $22(95.6)$ & $\mathrm{I}(4.3)$ \\
\hline $\begin{array}{c}\text { Vendas después } \\
\text { de cX }\end{array}$ & $22(95.6)$ & & $\mathrm{I}(4.3)$ \\
\hline $\begin{array}{c}\text { Medias antes de } \\
\text { cX }\end{array}$ & $2(8.6)$ & $20(86.9)$ & $\mathrm{I}(4.3)$ \\
\hline $\begin{array}{c}\text { Uso de } \\
\text { venoactivos }\end{array}$ & $3(13)$ & $19(82.6)$ & $\mathrm{I}(4.3)$ \\
\hline Uso de antibiótico & $16(69.6)$ & $6(26)$ & $\mathrm{I}(4.3)$ \\
\hline Cremas tópicas & $22(85.6)$ & & $\mathrm{I}(4.3)$ \\
\hline
\end{tabular}

\begin{tabular}{|c|c|c|}
\hline \multicolumn{3}{|c|}{ Tabla 4. Hallazgos con dúplex } \\
\hline $\begin{array}{c}\text { Tipo de reflujo y } \\
\text { severidad }\end{array}$ & Pacientes & $\%$ \\
\hline Mixto & 7 & 30.4 \\
\hline Moderado & 4 & \\
\hline Severo & 3 & \\
\hline Ninguno & 1 & 4.3 \\
\hline Superficial & 9 & 39 \\
\hline Moderado & 3 & \\
\hline Severo & 6 & \\
\hline Sin datos & 6 & 26 \\
\hline Total & 23 & \\
\hline
\end{tabular}

Los hallazgos en los dúplex preoperatorios disponibles en 17 pacientes $(73.9 \%)$ se describen en Tabla 4. La cirugía realizada se refiere en la Tabla 5.

El tiempo de cicatrización de la úlcera en promedio fue de 1.5 meses (0.59) y el de observación posquirúrgico de 2.84 meses. Once pacientes (47.8\%) tuvieron un cicatrización en menos de un mes, diez $(43.47 \%)$ en dos meses y uno (4,3\%) duró tres meses en cicatrizar. En vista de ello realizamos un cruce de variables para analizar este punto comparando cada uno de los grupos según el tiempo de cicatrización y las diferentes variables, en la Tabla 6 se observan los resultados. El tiempo (meses) promedio de seguimiento fue de 2.8 (1.8), 2.95 (1) y 3 respectivamente.

\begin{tabular}{|c|c|c|}
\hline \multicolumn{3}{|c|}{ Tabla 5. Cirugía realizada } \\
\hline Tipo de cirugía & Pacientes & $\%$ \\
\hline Varicosafenectomía derecha & 9 & 39 \\
\hline Varicosafenectomía izquierda & 12 & 52 \\
\hline $\begin{array}{c}\text { Varicosafenectomia izquierda y } \\
\text { ligadura de perforantes }\end{array}$ & 1 & 4.3 \\
\hline Sin datos & 1 & 4.3 \\
\hline Total & 23 & \\
\hline
\end{tabular}

\section{Discusión}

Encontramos curación del 100\% de los pacientes con promedio menor de tres meses; este hallazgo difiere de la literatura pues el reporte del 2006 refiere un $85 \%$. Consideramos que la discrepancia se puede atribuir al tipo de pacientes llevados a cirugía, seguramente con mayor compromiso del sistema venoso profundo o con enfermedad aterosclerótica asociada, casos que nosotros no consideramos candidatos a cirugía. En esta serie hay preponderancia de afección del sistema superficial. El tiempo de cicatrización es comparable con el reportado en este artículo. ${ }^{6}$ Decidimos además comparar los que tuvieron cicatrización en uno, dos y tres meses con las diferentes variables, sin encontrar diferencias. Cabe anotar que otro desenlace que debe medirse es la posibilidad de recidivas que no se pudo evaluar por el seguimiento corto. El estudio actual refuerza las publicaciones que muestran como aquellos con UV se benefician de la cirugía aun cuando no haya cicatrizado en el momento del procedimiento.

\section{Conclusiones}

El estudio actual confirma la utilidad de la cirugía y que el éxito radica en la adecuada elección del caso, en especial en los enfermos con repercusión hemodinámica severa por reflujo en el sistema superficial.

\section{Recomendaciones}

Se deben realizar estudios complementarios que analicen la mejoría en la calidad de vida de quienes se someten a cirugía de várices, discriminando la primaria 
Tabla 6. Análisis bivariado entre el tiempo de cicatrización y las variables

\section{TIEMPO DE CICATRIZACIÓN (meses)}

\section{VARIABLES}

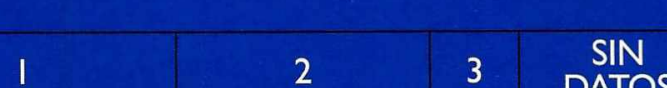

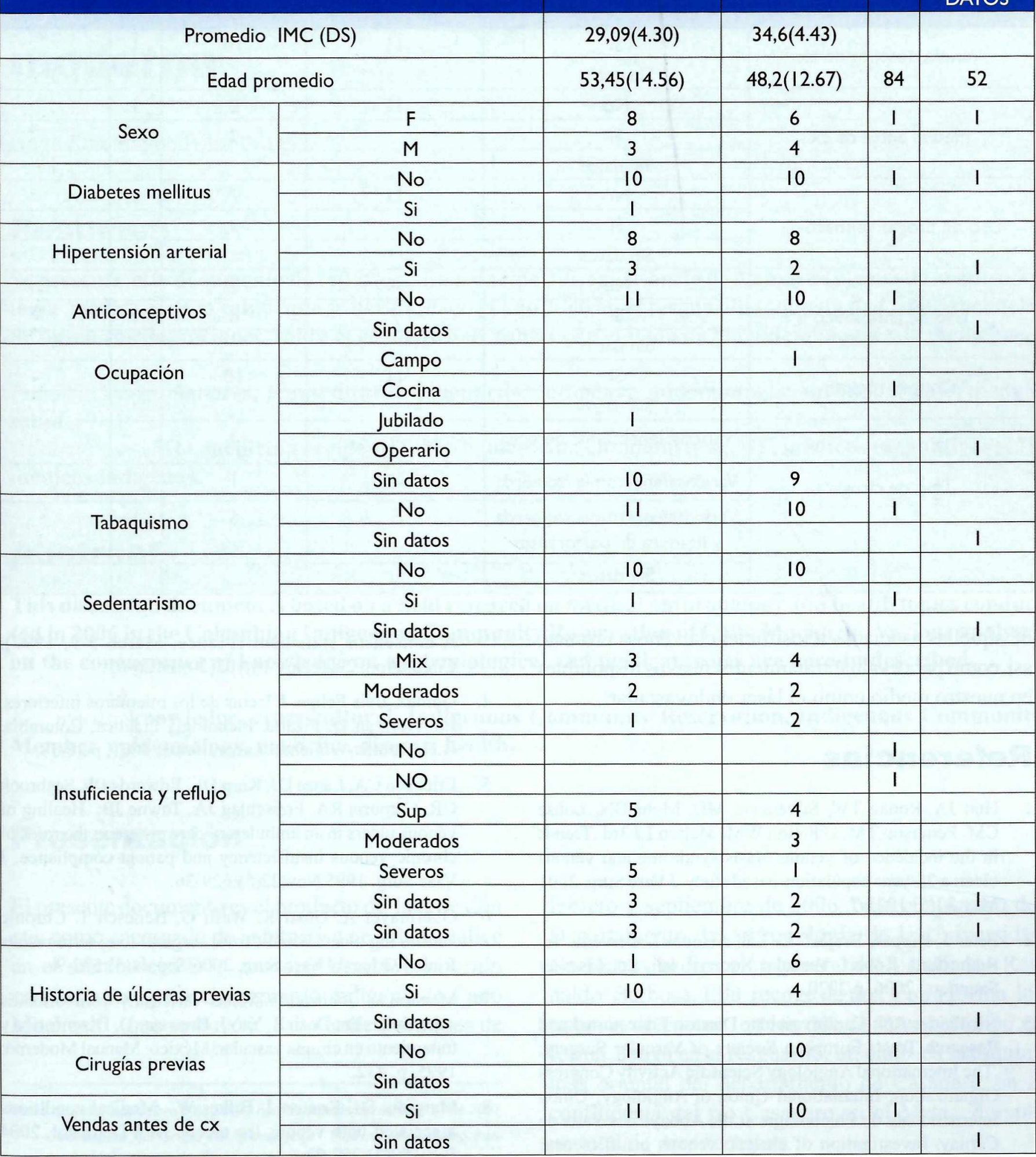




\section{Tabla 6. Análisis bivariado entre el tiempo de cicatrización y las variables (Continuación)}

\section{TIEMPO DE CICATRIZACIÓN (meses)}

\section{VARIABLES}

\begin{tabular}{|c|c|c|c|c|c|}
\hline & & I & 2 & 3 & DATOS \\
\hline \multirow{2}{*}{ Vendas después de cx } & $\mathrm{Si}$ & II & 10 & 1 & \\
\hline & Sin datos & & & & 1 \\
\hline \multirow{3}{*}{ Medias antes de cx } & No & 11 & 8 & 1 & \\
\hline & $\mathrm{Si}$ & & 2 & & \\
\hline & Sin datos & & & & 1 \\
\hline \multirow{3}{*}{ Uso de drogas venoactivas } & No & II & 7 & 1 & \\
\hline & $\mathrm{Si}$ & & 3 & & \\
\hline & Sin datos & & & & 1 \\
\hline \multirow{3}{*}{ Uso de antibiótico } & No & 4 & 2 & & \\
\hline & $\mathrm{Si}$ & 7 & 8 & I & \\
\hline & Sin datos & & & & 1 \\
\hline \multirow{2}{*}{ Cremas tópicas } & $\mathrm{Si}$ & 11 & 10 & 1 & \\
\hline & Sin datos & & & & 1 \\
\hline \multirow{4}{*}{ Tipo de cirugía } & Varicosafenectomía derecha & 6 & 3 & & \\
\hline & Varicosafenectomía izquierda & & 1 & & \\
\hline & $\begin{array}{c}\text { Varicosafenectomía izquierda } \\
\text { y ligadura de perforantes }\end{array}$ & 5 & 6 & 1 & \\
\hline & Sin datos & & & & 1 \\
\hline
\end{tabular}

de aquellas asociadas a insuficiencia venosa crónica, así como para evaluar tratamientos nuevos disponibles en nuestro medio como el láser endovascular.

\section{Referencias}

1. Heit JA, Rooke TW, Silverstein MD, Mohr DN, Lohse CM, Petterson TM, O'Fallon WM, Melton LJ 3rd. Trends in the incidence of venous stasis syndrome and venous ulcer: a 25-year population-based study. J Vasc Surg. 2001 May;33(5):1022-7.

2. Peter J. Pappas, Brajesh K. Lal, Joaquin J. Cerveira. In: Rutherford, Robert. Vascular Surgery. 6th. Ed. Elsevier Saunders; 2006. p 2220.

3. Nicolaides AN; Cardiovascular Disease Educational and Research Trust; European Society of Vascular Surgery; ,The International Angiology Scientific Activity Congress Organization; International Union of Angiology; Union Internationale de Phlebologie at the Abbaye des Vaux de Cernay. Investigation of chronic venous insufficiency:
A consensus statement (France, March 5-9, 1997). Circulation. 2000 Nov 14;102(20):E126-63.

4. Gómez, Luis Felipe. Ulceras de los miembros inferiores. En: Norman D. Pizano. Flebologia Práctica. Colombia. A.H. Robins-Whitehall Laboratorios; 2001. p 36-38.

5. Erickson CA, Lanza DJ, Karp DL, Edwards JW, Seabrook GR, Cambria RA, Freischlag JA, Towne JB. Healing of venous ulcers in an ambulatory care program: the roles of chronic venous insufficiency and patient compliance. J Vasc Surg. 1995 Nov;22(5):629-36.

6. Obermayer A, Göstl K, Walli G, Benesch T. Chronic venous leg ulcers benefit from surgery: long-term results from 173 legs. J Vasc Surg. 2006 Sep;44(3):572-9.

7. Marston W, Johnson G. Venas Varicosa y tromboflebitis superficial. En: Dean R, Yao J, Brewster D. Diagnóstico y tratamiento en cirugía vascular. México: Manual Moderno; 1995. p. 432.

8. Margolis DJ, Knauss J, Bilker W. Medical conditions associated with venous leg ulcers. Br J Dermatol. 2004 Feb;150(2):267-73. 